\title{
LA ATENCIÓN DE LA SOLICITUD ${ }^{1}$
}

\author{
Patricio Mena Malet ${ }^{2}$ \\ Universidad de La Frontera \\ patricio.mena.m@ufrontera.cl
}

\begin{abstract}
Resumen / ABSTRACT
El siguiente texto busca interrogar la dimensión atencional de la existencia humana a partir de los aportes realizados por Paul Ricœur en su obra fenomenológica temprana. Junto con ello, proponemos complementar la ética de la solicitud que el autor desarrolla en Soi-même comme un autre con una fenomenología de la atención que, a nuestro juicio, permite una comprensión enraizada en la experiencia humana de la que carece el análisis de la solicitud propuesto por Ricœur.
\end{abstract}

Palabras clave: Ricœur, atención, solicitud, disponibilidad, vigilancia ética.

\section{THE ATTENTION OF THE SOLICITUDE}

The following paper seeks to interrogate the attentional dimension of human existence from the contributions made by Paul Ricoeur in his early phenomenological work. Along with this, we propose to complement the ethic of solicitude developed by him in his main work Soi-même comme un autre with a phenomenology of attention. In our opinion, this could allow to obtain an understanding rooted in the human experience which is lacking in the analysis of solicitude proposed by Ricoeur.

KEYWORDS: Ricoeur, attention, solicitude, availability, ethical watch.

\section{Introducción}

D) Según Bernhard Waldenfels: "Es evidente que la atención no forma parte de los grandes temas de la filosofía occidental en tanto que aquella se ocupa del ser, del tiempo, del espacio, de la libertad o del sujeto. Una reserva tal da que pensar"

1 Este artículo presenta resultados de la investigación desarrollada en el marco del Proyecto Fondecyt Regular No 1160097 (2016-2018) del que el autor es coinvestigador.

2 El autor de este artículo quiere agradecer particularmente a los profesores Olivier Abel (Fonds Ricœur), Jean-Philippe Pierron (U. de Lyon), Jean-Luc Amalric (Fonds Ricœur), Natalie de Depraz (U. de Rouen) y Felipe Johnson (U. de La Frontera) de quienes, en distintos contextos, se ha beneficiado de las discusiones personales que ha podido mantener con ellos en torno a la atención, la vigilancia y la solicitud. 
(2010, p. 33). Así también Natalie Depraz constata el poco interés que ha despertado la atención en la filosofía contemporánea:

Husserl, afirma la autora, ha promovido una nueva concepción de la consciencia, Heidegger ha repensado el tiempo, Merleau-Ponty ha puesto en evidencia una filosofía de la carne, Levinas la alteridad. Difícil decir lo mismo tanto de la sorpresa o de la atención. ¿Qué filósofo invocar? ¿Dónde están los filósofos de la atención? ¿De la sorpresa? (2015, p. 261).

Y aunque es cierto que la atención no es particularmente de aquellas preocupaciones que más ha inquietado a los filósofos contemporáneos, no es menos cierto que las generaciones actuales de fenomenólogos se han volcado a examinar el fenómeno atencional en sus diversas dimensiones, a saber, epistemológica, ética, estética, entre otras ${ }^{3}$, viniendo de algún modo a realizar aquello que el propio Husserl tan solo bosquejó pero no realizó de modo acabado, a saber, una fenomenología de la atención ${ }^{4}$. Ante tal panorama cobra una vital importancia la reciente publicación del estudio de Paul Ricœur-originalmente publicado en 1940 - titulado, “L'attention. Étude phénoménologique de l'attention et de ses connexions philosophiques"5 (2013, pp. 51-93). En primer lugar, porque tratándose del primer trabajo fenomenológico publicado de nuestro autor, presenta ya una visión original respecto del tratamiento que le da Husserl a la atención. En segundo lugar, porque da cuenta del modo cómo Ricœur, desde esta época -diez años antes de la publicación de Le volontaire et l'involontaire (1950)-, intenta conjugar el método riguroso de la fenomenología que busca ir a las cosas mismas y retomarlas a partir de la descripción de sus estructuras esenciales de donación, con la filosofía de Gabriel Marcel que se aboca a pensar el misterio de nuestra existencia encarnada. Aquella estrategia de método, que Ricœur concretiza en Le volontaire et l'involontaire, ya era seguida por nuestro autor en el estudio fenomenológico sobre la atención de 1940, y gracias a la cual se precave de abordar el fenómeno atencional solamente en su dimensión epistémica y gnoseológica: la atención es pensada ya como un acto propio de la libertad, como expresión de la disponibilidad que el sí-mismo mantiene hacia la otra persona. He aquí

3 La fenomenología actual -y en ocasiones en cruce con las neurociencias- se ha abocado a la tarea de realización de una descripción de la atención relevando tanto su dimensión epistémica, responsiva (Waldenfels 2010, 2015), ética (Cf. Ricœur 2013; Steinbock 2010; Depraz 2014), intersubjetiva o conjunta ( $C f$. Bimbenet 2010; Depraz 2014), estética (Cf. Schaeffer 2015; Macié 2011 y 2016) o como movimiento de la vida subjetiva ( $C f$. Barbaras 2006, pp. 103-131). De esta manera, el interés creciente y reciente de parte de la fenomenología por la atencionalidad ha permitido nutrir y complejizar su descripción al reconocer a su vez que la atención no es tan solo una modulación regional o general de la vida intencional del sujeto, sino que ella posee un estatuto propio que vale la pena examinar.

4 Husserl que, habiendo dictado entre 1904 y 1905 un curso titulado Percepción y atención (Husserl 2009. Cf. Husserl 1998), no ha llegado sin embargo a proponer una fenomenología de la atención propiamente tal. Cf. Depraz 2008 y 2010.

5 Conferencia dictada originalmente en Rennes el 2 de marzo de 1939 en el Cercle philosophique de l'Ouest y publicada en el Bulletin du Cercle philosophique de l'Ouest en 1940. 
entonces lo que nos da que pensar: el fenómeno de la atención no solo tiene alcances gnoseológicos, sino que aporta elementos de comprensión respecto de lo que significa hacer el encuentro de la otra persona, orientarse y disponerse hacia ella. Siendo que Ricœur abrió esta vía de interrogación, y que amplifica a su vez una comprensión más vasta de la atención situándola en la base del encuentro ético con el otro, parece pertinente preguntarnos qué tipo de clarificaciones puede aportar una fenomenología de la atención, como la bosquejada por nuestro autor-pero que además pide prolongaciones por su estado más bien germinal-, a una ética de la solicitud, tal como la planteada por Ricœur en Soi-même comme un autre. Esto, en cuanto la expresión solicitud indica claramente que lo que está en juego es el cuidado del otro, esto es, la atención prestada hacia su persona. Y entonces parece muy claro que, entre la descripción eidética que Ricœur propone de la atención (Ricœur 2013, pp. 51-93; 1950, pp. 142-155)) -que toma sus distancias por lo demás de las realizadas por Husserl-y la comprensión que aporta respecto de la solicitud o cuidado del otro, hay una complementariedad que es preciso explorar y destacar, toda vez que el filósofo francés no lo hizo él mismo. A este respecto, el modo cómo el filósofo aborda la solicitud en tanto que "espontaneidad benevolente" (Ricœur 1990, p. 222) cuyas dimensiones estructurales son la reversibilidad (de roles), la insustituibilidad (de las personas) y la similitud (entre los sujetos en cuanto capaces), lo conduce a comprenderla muy rápidamente en el marco del "anhelo ético" que tiene como aspiración poder llevar una vida realizada con y para otros en instituciones justas (Cf. Ricœur 1990, pp. 202 et ss.). Mas, ¿no es preciso para comprender la solicitud como cuidado del otro, como comportamiento ético, esclarecer aquellas estructuras que le son propias y esenciales y que bien pudieran hallar su fundamento en la capacidad de orientarse atencionalmente del ser humano?

\section{Dos ámbitos propios de la solicitud}

La solicitud (sollicitude, Fürsorge; lat. sollicitudo) indica sin ambigüedades "cuidado", “inquietud”, "preocupación” y “atención”, pero también remite a la acción y efecto de solicitar, de demandar, de pedir, etc. Cuando Ricœur dice solicitud se refiere ciertamente al ámbito del cuidado por el otro. Pero, ¿el sentido de diligencia, de demanda, petición, puede aportar alguna claridad a la ética de la solicitud? Examinemos esta cuestión. Cuando en castellano se dice "solicitud" y se indica con ello la demanda que proviene del otro, ésta no necesariamente tiene un carácter ético; enseguida, cuando se dice de alguien que es "solícito" tampoco se dice en primera instancia que su respuesta tenga como fondo de su preocupación al otro, sino que quien responde lo hace con premura o que está dispuesto a responder a tal o cual demanda, que tiene por tanto cierta disponibilidad para ello. Así, la solicitud, en este sentido, subraya la dimensión respondiente de aquel que reconocemos como solícito -sin que ello ponga en juego necesariamente el carácter ético de la respuesta-así como su disponibilidad atencional: aquel que es solícito lo es porque en cierto contexto se mantiene atento a la demanda recibida como tal. Por otra parte, se puede indicar que las cosas mismas, que los asuntos en los que estamos implicados, nos solicitan. De esta manera, uno podría decir: "me siento solicitado por esto o aquello", y con ello se da cuenta de que estamos en relación 
de implicación con las cosas, los asuntos. Yo puedo comportarme solícitamente ante alguien, en cuanto por ejemplo respondo a una demanda específica que me es hecha o por una tarea que me es asignada o que yo mismo me doy, pero también puedo actuar solícitamente ante una situación específica que reconozco como demandándome en un ámbito delimitado de mi existencia social, afectiva, corporal, etc. Así, el acto de pedir o demandar refiere al cuidado, a la inquietud y a la atención, pues pone en juego al sujeto capaz de responder a la demanda, en cierto modo, atento a ella y capaz, a su vez, de reconocerse implicado por un ámbito, una situación, que abre la solicitud que viene $o$ de una persona $o$ de las cosas y asuntos mismos. De esta manera, es posible reconocer los ámbitos de la receptividad y de la respuesta ante el carácter solicitante de las cosas y de la otra persona, así como el de la atención a éste y la libertad que constituye nuestro responder. Que alguien sea solícito indica entonces que esa persona responde con premura $o$ a la demanda de alguien más, $o$ a una situación que de alguna manera lo exhorta; y entonces, ser solícito tiene como fundamento la capacidad del ser humano -se comporte o no solícitamente- de reconocerse inquietado, de preocuparse $o$ por sí mismo $o$ por otro, y de poder atender a eso que lo demanda. La solicitud, en este sentido, demanda el esclarecimiento de este modo de ser o estructura propia del ser humano capaz de sentirse solicitado. Así, la solicitud, en cuanto demanda o petición, permite poner el acento en lo que nos parece ser el fundamento de una ética de la solicitud: la capacidad del sujeto ético de reconocerse exhortado por la presencia de las cosas y de las personas, así como la capacidad de responder a ella. Alguien solícito es aquel que, en algún aspecto, hace de la demanda que le es dirigida, una inquietud propia que lo abre al camino del responder.

No resulta indiferente si Ricœur en algunas conferencias previas a la publicación de Soi-même comme un autre (Cf. Ricœur 1988; 1989), para referirse a la estima de sí, a la solicitud y a la institución, hiciera uso de la expresión souci (cuidado). Así, la tríada ética es designada como cuidado de sí, del otro y aquel propio de la institución. Y en efecto, la solicitud indica el cuidado por el otro. He aquí entonces lo que me interesa, y que a mi juicio puede fundamentar esta exploración que propongo: cuando se habla de "cuidado" (souci, care, etc.) también decimos "atención". Hay expresiones tales como: “¡ten cuidado con...!", "cuida bien a...”, “¡cuídate!”, que indican al menos dos cosas: 1) "presta atención a...", por ejemplo, al camino pedregoso, que si no lo haces te puedes caer, o, en otro sentido, "presta atención con" quien te relacionas, con quien haces amistad, etc.; 2) en los casos cuando le solicitamos a alguien que cuide bien, por ejemplo, a un hijo, o que se cuide a sí mismo, por ejemplo de no enfermarse, lo que le estamos pidiendo es que "preste atención a" otra persona o que preste atención a sí mismo; y en estos casos, atender o atenderse implica a su vez no solo un despliegue atencional tal como estar vuelto perceptivamente hacia otro o hacia sí mismo, sino también la capacidad de poder hacerse cargo de alguien o de sí, esto es, de considerarse y de ser responsable de sí y de otro. Podemos hallar más ejemplos en los que la "atención" remite al "cuidado" de sí o de lo otro, como cuando decimos "atiende esto o aquello", "préstale atención a tu hermano", etc. En todos estos casos, se puede reconocer una reciprocidad o reversibilidad entre cuidar y atender. Y con ello, habría que reconocer desde ya que el acto atencional sobrepasa la dimensión epistémica a la que es remitido. 
Atender no es solo un acto epistémico y gnoseológico, pues también es una suerte de cuidado de y por, una manera de ser responsable o de hacerse cargo de. Esta misma imbricación se puede hallar en expresiones inglesas tales como take care of someone (tomar cuidado de) o take care (poner atención).

Con todo, son estas indicaciones propias del lenguaje que aproximan y distancian a su vez al fenómeno de la solicitud al de la atención, las que me parece que justifican intentar interrogar de modo más acucioso sus relaciones y, por otra parte, complementar la ética de la solicitud, tal como fue elaborada por Ricœur, con una fenomenología de la atención que no se limite a abordar a esta última como un acto epistémico, sino que busque ahondar en su sentido pragmático y ético. Con ello, en mi opinión, es posible aportar mayor comprensión respecto de la solicitud, enraizándola en el existir encarnado que somos, reintegrándola así a la fenomenología del "yo puedo".

\section{La atención voluntaria del hombre actuante}

En la presentación que hacen Johann Michel y Jérôme Porée al tercer volumen de Écrits et conférences. Anthropologie philosophique de Paul Ricœur, los autores indican que su conferencia sobre la atención parece ser depositaria de una u otra forma de intuiciones filosóficas fundamentales del filósofo que se mantendrán a lo largo de su pensamiento, pudiendo tejerse un cierto hilo entre el "hombre atencional" y el "hombre capaz" ( $C f$. Michel y Porée 2013, pp. 13-14), tal como es pensado este último a partir de Soi-même comme un autre (1990) y Parcours de la reconnaissance (2004). Mas, lo que está en juego es algo más de fondo, en mi opinión, a saber, que el hombre capaz es, en primer lugar, un sujeto atencional o, dicho de otro modo, las capacidades humanas a partir de las cuales el sí-mismo despliega su acción en el mundo interviniendo en él con, por y para los otros, son ellas mismas un modo de despliegue y orientación atencional. Así, el atender no se restringe al poner atención propio de la percepción. De este modo, Ricoeur (Cf. 2013, p. 56) reconoce tempranamente "la existencia de una atención intelectual y de una atención perceptiva [que] confirma la extensión que debemos reconocer al percibir" (Ricœur 2013, pp. 56-57. El destacado es nuestro) ${ }^{6}$. Y de esta manera, nuestro autor identifica junto a la atención en la percepción -o percepción atenta-, una en el querer, en el poder, en suma, en el actuar; es así como en su conferencia del año 1939, Ricœur hablará de una atención voluntaria, siendo entendida como una orientación perceptiva y práctica, comprometiendo, por tanto, nuestra vida intencional y actuante. De este modo, las capacidades son ciertamente un modo de estar vuelto atencionalmente al mundo y a los otros. Mas, ¿qué puede significar aquello? ¿Qué puede significar que el hombre capaz, en tanto que capaz, esté atencionalmente dirigido hacia..., sino que

6 Aunque esta es una cuestión a la que el propio Husserl ya había atendido al examinar, por ejemplo, la atención en actos teóricos o afectivos tales como la admiración ( $C f$. Husserl 2009, pp. 103-105; Ricœur 2013, p. 70). 
las capacidades son un modo de orientarnos hacia las cosas como de hacernos cargo de ellas y de nosotros en ellas, de actuar sobre ellas y sobre nosotros?

La atención, como tal, es parte constitutiva del "yo quiero" y del "yo puedo" o del "hombre capaz", por las siguientes razones: 1) Aquella es pensada no solo como la dimensión atencional del ser humano en relación con el ámbito de la percepción, sino también con el de la voluntad -la deliberación (Cf. Amalric 2013, pp. 95-113), la elección y la decisión-, por lo que es parte constituyente de la conciencia teórica $y$ actuante. 2) Esto indica que la atención juega un rol de orientación y anticipación, pero sobre todo, de prospección y de resolución, sin el cual el cogito práctico no podría querer ni proyectarse ni tomar la iniciativa en el mundo. 3) La atención modula la vida intencional $y$ el despliegue de las capacidades prácticas, esto es, ella modula todo acto intencional, como la percepción, el recuerdo, la imaginación, etc., siendo que todo tender hacia, toda conciencia de... se despliega como una atención dirigida a..., y de esta manera se puede hablar, como lo hace Ricœur, de una percepción atenta, de un recuerdo atento, de un querer atento, etc. 4) La atencionalidad en la intencionalidad alcanza también a la conciencia actuante dirigida no solo a objetos mentados, sino también a los motivos y valores por los que actuar, así como a los posibles prácticos propios de las cosas queridas y actuadas. 5) Aquello también implica que la atención no recae solo sobre objetos intencionales, sino también sobre motivos, valores, que, de alguna manera, nutren y anticipan nuestro querer y nuestro poder ${ }^{7}$ y cuyo despliegue implica un esfuerzo constante por retomarse acogiendo el involuntario corporal -al que es posible oponer resistencia- y el involuntario absoluto del carácter, de la vida y del inconsciente -al que solo es dable consentir-. 6) Con todo, la atención no consiste solo en un acto de focalización perceptiva, de concentración y de clarificación a partir del cual un objeto es sacado de un segundo plano y conducido al primero, siendo realzado del horizonte perceptivo, sino que también ella es receptiva -antes que meramente pasiva-; pues atender también implica recibir, acoger, por ejemplo, aquellos motivos y valores sobre los que ella recae hasta decidirlos. 7) Estos caracteres aún preliminares de la atención -voluntaria y que es propia de la conciencia actuante- ya indican la posición de Ricœur respecto del sujeto, el sujeto de la atención: éste no es un ego constituyente de sentido, fundamento de éste y transparente para sí mismo, sino que es un cogito herido por la presencia del mundo, de las cosas y de los otros; es un cogito que se comprende en una relación inherente con la alteridad, por lo que la constitución de sí es una tarea

Al respecto, Jean-Luc Amalric afirma lo que sigue: "Del mismo modo que en la percepción la atención consiste en volverse hacia el objeto liberándolo de un trasfondo de la experiencia de modo de conferirle un relieve y una claridad especial, del mismo modo, en la deliberación y la elección, la atención se vuelve hacia los motivos y los valores con el fin de clarificarlos progresivamente. La atención a las cosas y la atención a los valores tienen en común desarrollarse en el tiempo y parece que se pueda igualmente establecer una analogía entre la donación en esquemas de la cosa que constituye la estructura de base de la percepción y la donación en escorzos de motivo y del valor en la decisión” (2013, p. 103). 
por ser conquistada en el encuentro con lo otro que demanda ser acogido, elegido y decidido. Y aquella relación con la alteridad que demanda atención-también en cuanto cuidado- tiene su fundamento en la propia experiencia corporal vivida como fuente nutriente de nuestro querer y como resistencia al querer y poder. Es en el seno de esta relación inherente, implicada, que vive el cogito herido con lo otro, su cuerpo propio y la situación en la que se halla, la que pone en juego la atención, en este ámbito de cuestiones, perceptiva pero también voluntaria.

Finalmente, es preciso relevar el hecho de que tanto la atención perceptiva-vuelta hacia los objetos- como la atención voluntaria - centrada en la captación de motivos, valores, etc.- comparten un mismo modo de interrogar lo atendido en cuanto éste-sea un objeto intencional o un valor-se da por escorzos, esto es, nunca se da de una sola y buena vez. La intención de la atención es la de aportar claridad y distinción respecto de los modos de aparición o donación de lo que se atiende, pero aquello exige que la atención sea una exploración interrogativa continua con el fin de poder ofrecer recursos imaginativos para decidir. Así lo explica Jean-Luc Amalric:

En la perspectiva de la decisión -afirma el autor-, si existe siempre una cierta tensión entre la vacilación (hésitation) y la elección es porque partimos de una confusión inicial de los diferentes valores y de una opacidad fundamental de nuestras motivaciones que es el hecho de nuestra existencia corporal. El rol de la atención es desde entonces aclarar y dominar una duración originariamente confusa. Del mismo modo que en la percepción, la atención es lo que da forma al objeto a partir de una materia impresional dada en esquemas, de la misma manera, en la decisión, la atención da forma a nuestro deseo a partir de una materia afectiva dada en esquemas (Amalric 2013, pp. 103-104).

De esta manera, la atención voluntaria, enraizada corporalmente, es vacilante, exploratoria y excedida por el hecho de que los valores y motivos -dados primeramente de modo confuso- remiten a posibles prácticos sobre los que hay que decidir para actuar. En este sentido, la atención voluntaria no solo busca aportar claridad ante lo atendido para que así la decisión zanje la vacilación en que consiste atender, sino que también orienta prospectivamente, atravesando al objeto de su atención para disponernos imaginativamente hacia los posibles por ser apropiados por la acción. Con ello, lo que quisiera destacar es que la atención deliberativa, por ejemplo, no solo está orientada en pos de la clarificación de lo que nutre la decisión y que podría implicar un movimiento retrospectivo hacia el arjé de nuestro querer, sino que también está vuelta hacia los posibles prácticos que orientan, configuran y refiguran nuestro actuar; esta orientación atencional es entonces prospectiva, antes que meramente anticipativa; pues no se trata aquí de anticipar el todo de lo parcialmente atendido, sino de quedar abierto y disponible hacia la captación, que no es sino la apropiación de una posibilidad práctica para la concreción de nuestro actuar. Esta cuestión me parece fundamental a la hora de interrogar la solicitud en su dimensión atencional. Mas para lograr mayor clarificación al respecto, examinemos en primer lugar dos modos propios de la atención: el hecho de que la atención implica, por un lado, el acto de poner atención, y por otro, que pueda ser suscitada. 


\section{Poner atención y suscitar la atención}

Para comprender dicho enraizamiento de la atención en la vida práctica -atención voluntaria-, que implica reconocer que la atención es tanto acto como recepción en la existencia actuante, vale la pena detenerse en la atención perceptiva. En efecto, cuando se interroga los modos de concreción de la atención se puede distinguir entre un "poner atención" (faire attention) y una "atención suscitada" o "inclinada" ( $C f$. Vincent 2015). En el primer caso, poner atención implica el libre despliegue del sujeto percipiente hacia los objetos, fijándolos, destacándolos, en suma, esclareciéndolos. Este movimiento atencional propio de la percepción lo es también de la atención intelectual, como cuando, en palabras de Ricœur: "mi atención puede desplazarse de la visión de una piedra a la consideración de una hipótesis, de [esta cosa] a una idea muy abstracta" (2013, p. 57). Lo interesante de esto, y que supone el reconocimiento de la extensión del percibir (Cf. Ricœur 2013, pp. 56-579) a actos intelectuales, signitivos, rememorativos, etc., es que en cada caso la atención implica la capacidad del sujeto para entrar en relación con objetos perceptivos, intelectuales, signitivos... Pero, al mismo tiempo, el acto de atender es un acto de distribución que hace pasar a los objetos de la latencia a la patencia, de un trasfondo a un primer plano, lo que pone en juego a su vez un "fenómeno de contraste entre figura y fondo" (Ricœur 2013, p. 59); y por ello, atender es remarcar, destacar, mas, siempre objetos. El acto de atender concierne por ende a un sujeto capaz de volverse intencionalmente hacia objetos constituidos por él. Además Ricœur agrega: 'la atención es una especie de acción; ella 'acentúa', 'hace surgir', en un sentido 'elige': hace aparecer algo del objeto. Pero -y es esta paradoja que constituye a la atención- la atención hace aparecer algo que en otro sentido ya estaba ahi" (Ricœur 2013, p. 62). ¿Qué significa esto? Lo que resulta interesante, en primer lugar, es que la atención dirigiéndose hacia las cosas, fijándolas y destacándolas, las hace aparecer en su calidad de objeto intencional, esto es, según el cómo de su manifestación, que por lo demás queda siempre abierto a otras modalidades de donación; con ello, la atención en algún sentido elige respecto de la cosa y su manifestación, esto es, conforme a las maneras que tenemos de atender, ella elige cómo aparecerá el objeto. La atención cobra entonces su sentido en tanto que acto intencional: tiene una función fenomenalizante que recae en el sujeto y los modos como se orienta atencionalmente hacia los objetos; pero, al mismo tiempo, la elección operada en el acto de atender-esto es, la concreción del poder de fenomenalización del atender- afecta al sentido de las cosas, su modo de donación, y no a las cosas mismas. Hay aquí dos cuestiones más que pueden ser relevadas: primero, la atención se dirige siempre primeramente hacia objetos, incluso si estos están en un segundo plano y son atendidos lateralmente, como cuando escuchamos una música de fondo sin que estemos orientados hacia ella, puesto que escuchamos lo que un colega nos dice; a lo más, se podría decir con Husserl que se trata de preobjetos o de objetos latentes, pero que siendo atendidos son también constituidos como objetos. Mas, y esto es relevante para Ricœur, en tal movimiento de destacamiento que hace pasar al objeto del trasfondo al primer plano, el objeto puede darse según un aspecto nuevo. Lo dado es lo mismo pero no termina de darse cuando lo percibimos, cuando lo atendemos de esta manera y no de aquella, cuando lo atendemos al recordarlo o cuando lo hacemos al pensarlo o desearlo; y en ese sentido, "la percepción nos da al existente 
como desbordando la percepción misma: el objeto -continúa Ricœur- es inagotable, puede ser continuamente rodeado según perspectivas en número indefinido" (Ricœur 2013, p. 64). En suma, el objeto atendido desborda nuestra experiencia atencional: "un objeto -dice el autor- no nos es dado nunca de un solo golpe" (2013, p. 64), aunque cada escorzo de la cosa la anuncie en su totalidad: lo que releva la anticipación propia del acto de atender.

Por otro lado, es interesante reconocer que Ricœur, conforme al modo como ha descrito el acto intencional, comprende que: "Nuestra exploración del mundo $-\mathrm{y}$ la experiencia de la identidad que la atraviesa- es una mezcla estrecha de cambios de aspectos que conduzco más o menos voluntariamente y de avatares que no dependen de mí" (Ricœur 2013, p. 66). Lo que resulta provocador es el reconocimiento que hace al interior del acto de atender - promovido por el sujeto que pone atención-como eso que depende del sujeto que lo lleva a cabo, pero también como no dependiendo del sujeto percipiente o queriente, por ejemplo. Y con ello se resalta el carácter pasivo o receptivo de la atención. Esto podemos repensarlo a través de la siguiente distinción: hay aquella atención que resulta del libre movimiento y orientación del sujeto, que es la que hemos parcialmente examinado y que identificamos con el poner atención, pero también hay una atención suscitada por la fuerza afectiva de las cosas. En este caso, la atención no tiene su iniciativa en el sujeto, sino que éste es movido, empujado a tomarla, por la presencia abrupta de un objeto que se impone y que haciéndolo, desvía la atención hacia él mismo. Si la atención implica el libre despliegue de la mirada hacia las cosas, que se enfoca en unas y luego en otras, también hay que reconocer que ella misma puede ser sorprendida y reorientada hacia aquello que no previó y que termina por imponerse desajustando o encontrando con la guardia baja al sujeto atento. Una atención sorprendida se reorienta a pesar de sí, aunque por sí. Esto es, cuando lo otro se impone, un ruido estruendoso o el color que resalta de un objeto o la vulnerabilidad del otro que nos toca y remece, el sujeto es desplazado del objeto de su atención; mas dicho desplazamiento es una posibilidad misma de aquel, a saber, la de poder ser despertado por y hacia las cosas mismas. ¿No encontramos aquí precisamente uno de los sentidos que tiene la expresión castellana "solicitud", es decir, el de ser demandado por otro o por las cosas mismas, lo que implica a su vez que ésta -la solicitud-nos introduce en un ámbito de cuestiones que podemos llamar concernientes?

En la atención suscitada, el sujeto se halla solicitado por las cosas a pesar de sí, lo que significa que un ámbito de interés se abre para él, y entonces mantiene la atención, o puede elegir, por el contrario, retornar a lo solicitante a un segundo plano para retomar la intención interrumpida y que lo ligaba a otra cosa que a ésta que se le había impuesto. Y de esta manera, la solicitación no quiebra la libertad de la mirada que atiende, pero la suscita de otro modo, la vuelve hacia el aspecto de las cosas que puede ser dicho, al menos en algún sentido, nuevo e inesperado. O, como ya lo he indicado, la atención es sorprendida con la guardia baja. Este punto me parece que es muy relevante. ¿En qué sentido ser alcanzado por la fuerza afectiva de las cosas, ser despertado hacia ellas, implica ser descubierto con la guardia baja? Pues, en efecto, cuando estamos orientados hacia esto o aquello nos hallamos de alguna forma implicados en una situación: estamos escribiendo una conferencia, por ejemplo, y todo nuestro compromiso atencional está 
vuelto hacia nuestros pensamientos y la expresión de aquellos que fijamos escribiéndolos; hasta que un grito de auxilio nos saca de la situación y entonces actuamos y vamos en ayuda de quien lo solicita -sin que necesariamente se dirija a nosotros-. De pronto, cambiamos radicalmente de actitud, asumimos una postura corporal muy distinta a la que teníamos cuando escribíamos y saltamos de nuestro escritorio para correr al auxilio del afligido. Ciertamente, no era parte de nuestra expectativa ser solicitados como lo fuimos mientras estábamos hundidos en la situación intelectual en la que nos hallábamos. El grito se ha impuesto de tal modo que ha ocupado el primer plano de nuestro atender, y esto a nuestro pesar o sin que lo pudiésemos prever; y con ello, ha hecho caer todo aquello que era parte de mi atención inicial: mis pensamientos, el teclado, la pantalla, el escrito que estaba redactando, etc. Mas, y a pesar de ello, se podría objetar que se ha actuado solícitamente, con prontitud, y entonces no nos ha encontrado con la guardia baja. Pero, ésta no consiste, sin embargo, en la incapacidad de actuar, de responder a una solicitación, sino en la imposibilidad de no atender. La atención puede ser suscitada, puede ser sorprendida, porque ella es una disposición apertural continua. Así, mientras nos dirigimos hacia esto o aquello, intencionalmente y con intención, quedamos siempre abiertos hacia el encuentro con lo otro; y aquello no se da siempre por nuestra iniciativa: las cosas también nos encuentran, incluso, a pesar nuestro. Claro, la solicitación de las cosas depende de nuestra disponibilidad para ellas. Las cosas en sí no hablan, pero llaman conforme a los intereses con que nos orientamos en el mundo y nos dejamos concernir por aquel.

Entonces, lo propio de esta disponibilidad es ser una aperturidad fundada en la fragilidad que es estructuralmente constitutiva del ser humano. La lección que nos da Ricœur en L'homme faillible es ejemplar en este sentido. La fragilidad alcanza al ser del ser humano; y si, por un lado, éste es apertura, al mismo tiempo es perspectiva; si anida en él el deseo de una libertad total, al mismo tiempo, ésta no puede desplegarse sin hallarse enraizada corporalmente en el mundo, sin encontrar también la oposición de los otros, de las cosas, etc. Así, la disponibilidad apertural es frágil por su enraizamiento corporal y mundano; siempre somos despertados a... estando ya en una situación particular y no pudiendo atender tampoco a la sobrevenido de un modo pleno y total; pero también, y por ello mismo, la atención adquiere un carácter interrogativo $(C f$. Ricœur 2013, p. 69) y exploratorio en relación con el objeto dado que se impone. Que nos encuentre con la guardia baja significa que hace virar nuestra atención, pero también que la hace vacilar y que sea necesario que ésta recomience, que se apreste otra vez a la clarificación de lo dado. Pues, incluso en el ejemplo del grito de auxilio, y aunque solícitamente saltemos de nuestro escritorio, lo hacemos interrogándonos "¿qué habrá pasado?”, “¿cuál será la situación que hallaremos?”. No solo somos atraídos atencionalmente hacia el objeto que se impone, sino que el modo como quedamos vuelto hacia él es vacilante, inquietado e interrogativo. De algún modo, la mirada atencional es confrontada a su propia ingenuidad atendiendo sin saber exactamente a qué atendemos. En este sentido, la atención es prospectiva, pues vuelve hacia lo dado sin esperar que éste aparezca delimitadamente: ciertamente, se da, pero queda un trasfondo ensombrecido que antes de su dilucidación pide nuestra acción. De este modo, Ricœur afirma lo siguiente: 
Yo soy tanto más atento en cuanto busco menos 'cumplir' intuitivamente una intención vacía y más exploro ingenuamente el campo de percepción; no es la prepercepción ni el deseo que constituyen a la atención, sino la ingenuidad de la mirada, la inocencia de la mirada, la acogida de lo otro en tanto que otro. Por esta activa disponibilidad, yo me inscribo en la consideración del objeto. El verdadero nombre de la atención no es anticipación sino sorpresa; es lo contrario de la precipitación y de la prevención (1950, p. 147).

Una atención suscitada no es necesariamente precipitada pero sí solicitada. No somos despertados a las cosas sin que podamos decidir si damos libre curso a nuestra reorientación hacia ellas.

\section{Estar atento y ser atento: la vigilancia ética}

Hasta aquí, lo que me ha interesado examinar, con el fin de aportar recursos para la comprensión de la solicitud ética -en tanto que atención y cuidado- ha sido lo siguiente: mostrar que la atención es una modulación de todo acto intencional, incluida la voluntad; que está enraizada por consiguiente en nuestra vida corporal y mundana; que en ella nos volvemos hacia los objetos, motivos, valores y posibles prácticos, aunque siempre de un modo frágil que exige a la atención persistir en la manera como explora e interroga las cosas; por último, la atención no es un acto que esté del todo en nuestro poder, sino que ella también está constituida por lo que no depende del sujeto atencional, así por ejemplo pasa con la atención en su disponibilidad para ser suscitada. Habiendo realizado todas estas consideraciones quisiera ahora interrogar directamente la relación entre atención y solicitud examinando el vínculo que hay entre "estar atento" y "ser atento".

Ambas expresiones, "estar atento" y "ser atento", cuyos correlatos en francés son "être attentif" y "être attentionné", nos sitúan ya en el ámbito ético de la solicitud. Así, "estar atento" dice algo más que "prestar atención" en tanto acto que viene a remarcar el objeto intencional, a prestar un interés en él que implica una focalización y concentración que saca al objeto del trasfondo de latencia en la que se hallaba. "Estar atento" indica una espera vigilante, una disposición que se mantiene a la expectativa de recibir, de acoger, lo que no necesariamente puede ser previsto ni anticipado, o lo que no puede ser claramente tematizado, totalizado o dominado como lo es un objeto, sea intelectual, científico o un útil. Así, "estar atento" implica el acto de mantenerse atento, lo que a su vez conlleva cierto conatus y persistencia propio del atender y cuenta con una mirada sostenida en la ingenuidad como capacidad para descubrir lo que se dona según el modo de la primera vez. Es en este sentido que, a juicio de Ricœur, "el verdadero nombre de la atención no es anticipación sino sorpresa (étonnement)" (2013, p. 70; Cf. Depraz 2015). Ciertamente, es preciso reconocer que hay un modo de mantenerse atento que responde a la lógica de lo estereotipado, de lo típico, de lo disponible -del objeto en su disponibilidad-, y que es por ejemplo la atención del experto o del funcionario que se mantienen atentos para asegurar la continuidad, la invariabilidad de la producción; mas, lo que es verdaderamente propio de la atención, según nuestro autor, es la sorpresa, esto es, la capacidad de mantenerse abierta a lo 
que no se espera, a lo que no se prevé, es decir, a lo que no se reduce a objeto. O dicho de otra manera, hay un modo de "estar atento" que implica, por un lado, un descuido de sí en pos del cuidado atento a lo otro; o, si se quiere, esta atención mantenida se sostiene en una disponibilidad radical hacia lo otro que se traduce en una distracción profunda de sí a partir de la que el sujeto que atiende se halla abierto al encuentro, al contacto con lo atendido, sin que éste sea reincorporado tan rápidamente en la relación intencional objetivante. Y entonces, mantenerse abierto atencionalmente a lo otro es a su vez distraerse de sí. Así, entonces, la atención, manteniendo su libertad de exploración y su apertura ingenua hacia las cosas, consiente también la presencia y el advenir de lo otro sin fijarlo de antemano en una identidad que lo clausure. Y en tal sentido, si el verdadero nombre de la atención es la sorpresa habría que indicar que ésta se despliega conforme a cierta despragmatización ( $C f$. Schaeffer 2015, p. 71): estar atento es mantenerse abierto a su objeto sin presuponer de antemano su acceso, esto es, sin saber anticipadamente cómo acceder a él, cómo interrogarlo, pero tampoco sin buscar tematizarlo ni acotarlo de modo definitivo. Cuando atendemos al otro, al prójimo, por ejemplo, ¿no está en juego precisamente este modo de estar atento? Si, ciertamente, el otro no se presenta al modo como lo hacen las cosas, los objetos, los útiles, y si tampoco nos solicita de la misma manera, ¿no habría que indicar que la atención que requiere, la atención por la que nos es dado, es aquella cuyo verdadero nombre es la sorpresa, cuya mirada es ingenua y cuyas pretensiones no radican en totalizar ni objetivar al -paradójicamente- objeto de su atención?

Pero ahora es preciso destacar otro carácter del "estar atento" que puede alumbrar el fenómeno ético de la solicitud, i.e., la vigilancia. Según Natalie Depraz, ésta significa lo siguiente:

El término de 'vigilancia' ha sido recogido más arriba liberando a la atención de su componente racional (focal, funcional) y abriéndola a su cualidad de apertura receptiva y de espera sin objeto [...]. Etimológicamente, 'vigilancia viene del latín vigilare $[. .$.$] . Designa de modo corriente una vigilancia (surveillance) atenta \sin$ desfallecimiento. Más adelante, el vínculo directo con la vigilia indica una actitud de presencia del otro (vigilar a o por alguien), es decir, de cuidado por el otro, o aún un modo de presencia en vínculo directo con una espera, una cualidad tenue y ligera de paciencia, de inscripción en una duración vivida (Depraz 2014, p. 48).

La vigilancia es entonces la de atención hacia el otro (cuidado del otro) que no desfallece, esto es, que incluso desfalleciendo persiste en mantenerse ligada y atenta al otro; indica, con toda claridad, que vigilar es un modo de asumir el cuidado de la otra persona, i.e., un modo de ocuparse del otro de manera atencional que es persistente: la vigilancia es por ello conatus. No es solo el acto de atender, sino el de persistir en la atención. Si esta última, la mayor parte del tiempo, no requiere esfuerzo pues se orienta tan fácilmente sobre unas cosas para luego dirigirse a otras, la vigilancia es una atención al otro que compromete un esfuerzo: el de mantenerse alerta y despierta. Así pasa, por ejemplo, con los cuidados prodigados a un enfermo que en ocasiones implican desvelos, sobresaltos, inquietud constante ante la condición frágil de aquel, o con la espera inquieta de un ser amado que mantiene al amante expectante respecto de su eventual arribo. De 
esta manera, la vigilancia o la vigilia parece contener de un modo ejemplar el triple sentido de cuidado: inquietud, atención y ocupación -sobre sí y sobre el otro-. Es de esta forma, entonces, que la ética de la solicitud se halla enraizada en la vigilancia como los modos a partir de los cuales el ser humano se mantiene en vigilia, en vela y en vilo - expectante-, por el otro. ¿Cuáles son los elementos esenciales de la atención que constituyen su impulso ético y que pueden permitirnos dilucidar en qué sentido la solicitud -en tanto que cuidado del otro- debe ser comprendida como vigilancia atencional? Ciertamente, la vigilancia desmedida puede volverse fundamento de prácticas de control al interior de las instituciones, prácticas totalitarias o discriminatorias como cuando un país se mantiene atento a supuestas amenazas terroristas. Pero es el riesgo constante que corre la atención: quererse en conformidad al objeto y la objetividad, o hacer de la atención de sí la medida de todas las cosas, y entonces la atención repulsa lo otro como amenazante.

Mas, a pesar de los riesgos de una fenomenología de la atención que transita hacia una ética de la solicitud, la atención también vive la desmedida, el exceso, de modo positivo. Es el caso por ejemplo del "ser atento" (être attentioné). Cuando en castellano se dice "ser atento" se explícita con ello que la atención está volcada hacia la persona. Solo puedo ser atento con alguien y no con las cosas: a estas últimas, les presto atención, pero no soy atento con ellas; puedo prodigarles cuidados, ser solícito ante las cosas o asuntos que me conciernen, pero no "ser atento" con ellas, al menos no en un sentido propio. Así, cuando se dice que alguien "es atento" más bien se refiere con ello a cierta disposición positiva para ocuparse de la otra persona manteniéndose vigilante respecto de ella, de sus necesidades, requerimientos y solicitud. Compromete por tanto: 1) una orientación atencional, un estar atento a... y una atención suscitada por la presencia de la otra persona que nos solicita, por ejemplo, en su vulnerabilidad; 2) un compromiso práctico hacia aquel que nos solicita, esto es, comprometernos con acciones que respondan a su persona; 3) que las respuestas que le dirigimos a la otra persona no tienen por finalidad el esclarecimiento de un objeto, el destacamiento de aquel, sino verdaderamente responder a su solicitud; 4) "ser atento" implica una dialéctica entre cuidado del otro y descuido de sí; así, la atención puesta y en obra con respecto al otro requiere un cierto olvido de sí de parte de quien se muestra atento ante la presencia de la otra persona: "ser indisponible -afirma Ricœur- es estar ocupado en si" (1992, p. 71); y entonces, ser disponible, mantenerse disponible, es estar vuelto atencionalmente hacia el otro antes que a sí, aunque ello, por cierto, implique a su vez un esfuerzo por conocerse a sí en las capacidades a partir de las cuales nos es posible ocuparnos del otro.

Habría entonces que indicar que "ser atento", antes de designar un trato gentil respecto de la otra persona, indica un comportamiento, en vigilia, que se funda en la disponibilidad apertural del sujeto, en tanto que modo de ser del existir mismo a partir del que el ser humano se halla allí en su afuera, afuera de sí y en relación con lo otro. $\mathrm{Y}$ entonces, el sujeto atento da cuerpo y encarna en un conjunto actitudinal aquella disponibilidad apertural volviéndola una manera de estar abierto vigilantemente hacia el otro. ¿Por qué vigilantemente? Pues, la atención, que aquí está en juego, no solo da cuenta de la preocupación y ocupación por la presencia del otro, su necesidad y 
vulnerabilidad, sino que también nos dice que ser atento es una disposición que persiste o quiere persistir en tal modo de estar abierto. La vigilancia es entonces una atención enfocada que busca abrir a lo otro, pero, y por lo mismo, es una atención persistente puesto que aquello a lo que nos abre no concluye de darse o se da según un modo de ser continuo e indefinido. Es en ese sentido que "ser atento" implica un modo de ser vigilante ante la presencia de la otra persona y que busca los medios para no desfallecer. De esta manera, si la disponibilidad atencional aquí en juego implica un cierto olvido de sí, a su vez, conlleva una atención a sí en pos del mantenimiento de la atención al otro. Si la vigilancia en su modo de entrar en relación con el otro busca no decaer atencionalmente, entonces es preciso indicar que ella se sostiene en un tipo de atención a sí como un tenerse en cuenta para sostenerse alerta al otro: dicha atención a sí, cuidado de sí, ocupación de sí, halla su fundamento en la solicitud exhortante de la otra persona. No se trata de una atención que corre el peligro de volverse autocentración, sino de una reflexividad que tiene sentido - esto es, que está orientada (sentido-orientación)- en cuanto atiende con el fin de mantener y sostener la relación con el otro que nos requiere. Y entonces, la atención a sí es un modo de asegurar aquella manera de respuesta solícita hacia quien nos convoca.

\section{Conclusiones}

Cuando Ricœur describe la solicitud como espontaneidad benevolente descuida la descripción del cuidado y la atención al otro en su carácter más concreto, fundado en la experiencia. Así, a mi juicio, una comprensión honda de la solicitud ética llama, sin lugar a dudas, al despliegue de una fenomenología de la atención, que es lo que aquí he intentado. Si la solicitud es el cuidado que ponemos en el otro, era preciso explicar lo que implica atender a la otra persona en el trato cotidiano -y cuál es el fundamento de dicho atender-, y ciertamente dicha atención no puede quedar restringida como modulación de la intencionalidad perceptiva. Así, el primer avance promovido por Ricœur es el haber reconocido el vínculo estricto entre atención y libertad, y con ello haber inscrito la atención en la vida práctica del ser humano. Por otra parte, y nuestro autor nos ha prestado útiles conceptuales para ello, la atención no es solo un acto que dependa de un sujeto soberano o dueño de sí y de sus actos, sino que implica también una vida pasiva: el hombre atencional está abierto a las cosas de modo tal que es despertado a ellas por ellas. La atención suscitada da cuenta de esta disposición apertural que promueve un despliegue libre e ingenuo por las cosas. Mas, dicha libertad e ingenuidad no es sinónimo de desinterés, sino el fundamento del interés que podemos prestar -hasta hundirnos en él- en el trato que mantenemos con las cosas y con las otras personas. He aquí que se deja comprender la ligazón entre "estar atento" y "ser atento", que nos abre, a su vez, a las consideraciones del lugar que tiene la atención en el trato ético mantenido con los otros. Si en la distinción establecida entre "poner atención" y "suscitar la atención" la dialéctica en juego era la de la actividad y de la pasividad, aquello que se da a destacar en el lazo entre "estar" y "ser atento" es la persistencia que toma la forma de la vigilancia ética: estar y ser atento -que también moviliza la dialéctica entre actividad y pasividad-acentúa más bien el esfuerzo querido 
de mantener la atención con respecto a lo atendido, reconocido como lo que solicita y requiere respuestas, afrontamiento, ocupación. El hombre atencional, vigilante, que quiere no desfallecer ante la presencia lo hace a su vez reconociendo su indigencia, su ignorancia, su falta de respuesta adecuada. Así, la solicitud, como cuidado del otro, que debe ser complementada con una fenomenología de la atención, se deja comprender por la explicitación de la solicitud como demanda, petición: o si se quiere, el cuidado por el otro requiere la comprensión de los modos como el sujeto se moviliza hacia la otra persona e inicia así el camino de su respuesta.

\section{Referencias bibliográficas}

Amalric, Jean-Luc (2013), Paul Ricour, l'imagination vive. Une genèse de la philosophie ricœurienne de l'imagination. París: Hermann; 686 pp.

Barbaras, Renaud (2006), Le désir et la distance. Introduction à une phénoménologie de la perception. París: Vrin; 175 pp.

Bimbenet, Etienne (2010), "Pour une approche phénoménologique de l'attention conjointe", Alter. Revue de phénoménologie 18: 93-110.

Depraz, Natalie (2008), “Attentionalité et intentionnalité: l'attention comme 'modulation'. (Où est la phénoménologie de l'attention que Husserl avait le projet de mettre en oeuvre?)", Jocelyn Benoist (editor), Husserl. París: Cerf; pp. 223-247.

(2010), “Attention et conscience: à la croisée de la phénoménologie et des sciences cognitives", Revue de phénoménologie 18: 203-226.

(2014), Attention et vigilance. À la croisée de la phénoménologie et des sciences cognitives. París: PUF; 528 pp.

(2015), “Attention et surprise. Paul Ricœur en débat et au-delà", Alter. Revue de phénoménologie 23: 261-278.

Husserl, Edmund (1998), De la synthèse passive. Logique transcendantale et constitutions originaires. Grenoble: Jérôme Millon; 431 pp.

(2009), Phénoménologie de l'attention. París: Vrin; 258 pp.

Macié, Mariel (2011), Façons de lire, manières d'être. París: Gallimard; 288 pp.

(2016), Styles. Critique de nos formes de vie. París: Gallimard, 358 pp.

Michel, Johann y Jérôme Porée (2013), "Présentation", Paul Ricœur, Écrits et conférences 3. Anthropologie philosophique. París: Seuil; pp. 7-18.

Ricœur, Paul (1950), Philosophie de la volonté 1. Le volontaire et l'involontaire. París: Aubier; 465 pp.

(1988), "Quelle éthique en politique?", Supplément au Bulletin du Centre Protestant d'Études et de Documentation 334, III-VI.

(1989), "L'éthique, la morale et la règle", Autres Temps. Les cahiers du christianisme social 24: 52-59.

(1990), Soi-même comme un autre. París: Seuil; 428 pp. 
(1992), "Entre éthique et ontologie: la disponibilité", Lectures 2. La contrée des philosophes. París: Seuil; pp. 68-78. (2004), Parcours de la reconnaissance. Paris: Stock, 387 pp.

(2013), “L'attention. Étude phénoménologique de l'attention et de ses connexions philosophiques", Écrits et conférences 3. Anthropologie philosohique. París: Seuil; pp. 51-93.

Schaeffer, Jean-Marie (2015), L'expérience esthétique. París: Gallimard; 366 pp.

Steinbock, Anthony (2010), "Exemplarité, émotions et attention", Alter. Revue de phénoménologie 18: 59-75.

Vincent, Gilbert (2015), Hospitalité: la naissance symbolique de l'Humain. Estrasburgo: Presses Universitaires de Strasbourg; 371 pp.

Waldenfels, Bernhard (2010), "Attention suscitée et dirigée", Alter. Revue de phénoménologie 18: 33-44.

Waldenfels, Bernhard (2015), Exploraciones fenomenológicas acerca de lo extraño. Barcelona: Anthropos - Siglo XXI Editores; 333 pp. 